\title{
Prevalencia de hipocalcemia postiroidectomía en cirugía de cáncer de tiroides
}

\author{
Prevalence of Post-Thyroidectomy Hypocalcemia in Thyroid Cancer \\ Surgery
}

Mejía $M G^{1}$; Vega $M P^{2}$; Hakim JH

${ }^{1}$ Endocrinóloga, Departamento de Medicina Interna, sección de Endocrinología, Hospital Universitario Fundación Santa Fe de Bogotá, Colombia. Profesora Universidad de los Andes

${ }^{2}$ Médica, Universidad de los Andes, Facultad de Medicina. Bogotá, Colombia

${ }^{3}$ Cirujano de cabeza y cuello, Departamento de Cirugía, Hospital Universitario Fundación Santa Fe de Bogotá. Bogotá, Colombia.

Autor de correspondencia: marigmej@gmail.com

Fecha de recepción: 31/07/2018

Fecha de aceptación: 15/12/2018

\section{Resumen}

Introducción: La complicación más frecuente de la cirugía de cuello en cáncer de tiroides es el hipoparatiroidismo posoperatorio. Su prevalencia depende de muchas variables y sus repercusiones en calidad de vida son significativas. A la fecha no se conoce la prevalencia de hipoparatiroidismo posquirúrgico en cirugía de cáncer de tiroides en la población colombiana, haciendo que en nuestro país no se disponga de estrategias de prevención y manejo temprano.

Se presenta un estudio que muestra la prevalencia de hipocalcemia transitoria y permanente debida a hipoparatiroidismo postiroidectomía en una institución de cuarto nivel, utilizando un protocolo de suplementación de calcio y calcitriol en el posoperatorio inmediato de la cirugía como base para crear estrategias de prevención y manejo temprano en nuestros pacientes.

Métodos: Se realizó un estudio retrospectivo observacional tipo corte transversal con información obtenida de historias clínicas de pacientes sometidos a tiroidectomía por cáncer de tiroides entre los años 2003 y 2017 en la Fundación Santa Fe de Bogotá. Se recolectaron los datos a través del registro de base de datos de cirugía de cabeza y cuello. Se dividieron los grupos para evaluar prevalencia de hipocalcemia transitoria y permanente.

Resultados: Se analizaron los datos de 261 pacientes, encontrando una mediana de edad de 48,6 años. El análisis univariado evidenció mayor prevalencia de la enfermedad en mujeres $(75 \%)$ y el diagnóstico de carcinoma papilar de tiroides (85\%) fue el más relacionado a intervencion quirúrgica. La prevalencia de hipocalcemia posquirúrgica a los tres meses POP fue del $18 \%$, frente a $7 \%$ a los siete meses.

Conclusiones: La prevalencia de hipoparatiroidismo postiroidectomía para manejo de cáncer de tiroides en una institución de cuarto nivel de complejidad de Bogotá, en manos de un cirujano experto de alto volumen es cercana al 20\% con reducción al 8\% a los siete meses, con tasas menores a las reportadas pero significativas, lo cual demuestra la importancia de crear estrategias para prevención y manejo temprano. Existe la necesidad de registros a nivel nacional que permitan analizar el comportamiento en general de los pacientes sometidos a cirugía del compartimento central del cuello, con el fin de establecer protocolos en la suplementación que prevengan el porcentaje de morbilidad de los pacientes con secuelas permanentes.

Palabras clave: hipoparatiroidismo posquirúrgico, hipocalcemia posquirúrgica, Cirugía de cuello.

\section{ABSTRACT}

Background: The most frequent complication of neck surgery in thyroid cancer is postoperative hypoparathyroidism. Its prevalence depends on many variables and the repercussions on the quality of life of patients are significant. To date, the prevalence of postoperative hypoparathyroidism in thyroid cancer surgery in the Colombian population is unknown, interfering with early prevention and management strategies in our country.

We present a study that shows the prevalence of transient and permanent hypocalcemia due to post-thyroidectomy hypoparathyroidism in a 4th level institution, using a protocol of calcium and calcitriol supplementation in the immediate postoperative period of surgery as a basis to create prevention strategies and early management in our patients.

Methods: A cross-sectional, observational retrospective study was conducted with information obtained from clinical 
records of patients undergoing thyroidectomy for thyroid cancer between 2003 and 2017 at the "Hospital Universitario Santa Fé de Bogotá". Data were collected through the head and neck surgery database registry. The groups were divided to assess the prevalence of transient and permanent hypocalcemia.

Results: Data from 261 patients were analyzed, finding a median age of 48.6 years. The univariate analysis showed a higher prevalence of the disease in women (75\%) and the diagnosis of papillary thyroid carcinoma (85\%) was the most related to surgical intervention. The prevalence of post-surgical hypocalcemia at 3 months POP was 18\%, compared to $7 \%$ at 7 months.

Conclusions: The prevalence of post-thyroidectomy hypoparathyroidism for the management of thyroid cancer in a 4th level institution of complexity in Bogotá, in the hands of a high volume expert surgeon is close to $20 \%$ with reduction to $8 \%$ at 7 months, with rates less than those reported but significant, which demonstrates the importance of creating strategies for prevention and early management. There is a need for registers at a national level to analyze the behavior of patients undergoing surgery in the central compartment of the neck, in order to establish supplementation protocols that prevent the morbidity rate of patients with permanent sequelae.

Keywords: post-surgical hypoparathyroidism, post-surgical hypocalcemia, neck surgery.

\section{Introducción}

El hipoparatiroidismo es una patología infrecuente, que se caracteriza por hallazgos clínicos y paraclínicos consistentes con niveles bajos de calcio sérico, fósforo sérico alto y valores disminuidos o ausentes de $\mathrm{PTH}^{(1)}$. Son diversas las etiologías de la enfermedad, siendo el $75 \%$ de los casos secundarios a cirugía de cuello. Aunque depende de la institución donde se realicen las intervenciones, se ha descrito que hasta el 50\% de los pacientes posquirúrgicos pueden desarrollar hipoparatiroidismo transitorio y de $1 \%$ a $33 \%$ lo hacen de forma permanente ${ }^{(2,3)}$. Otras causas de hipoparatiroidismo son las enfermedades autoinmunes que afectan las glándulas endocrinas y, en menor medida, se describen desórdenes infiltrativos de las paratiroides o incluso enfermedades metastásicas ${ }^{(4)}$.

La presentación clínica es muy variable y la correlación está dada por la etiología de la enfermedad. Los signos y síntomas en pacientes con hipoparatiroidismo posquirúrgico son diferentes de los presentados por enfermedades autoinmunes o infiltrativas ${ }^{(4,5)}$. El estado ácido-base de la sangre, los bajos niveles de magnesio y la hiperactividad simpática determinan también la aparición de los síntomas ${ }^{(6)}$. Cuando la hipocalcemia es aguda y súbita existe un mayor compromiso del sistema neuromuscular, neuropsiquiátrico y cardiovascular. Estos cuadros pueden llegar a ser tan graves que requieran hospitalización, en especial por el riesgo de laringoespasmo, excitabi- lidad neuromuscular con disrupción sensitiva y motora, deterioro cognitivo y trastornos de la personalidad ${ }^{(7)}$. El espectro entre desarrollar los síntomas o ser asintomático depende del paciente y por supuesto de los valores séricos de calcio, junto con la rapidez y descenso de este electrolito. Los rangos de calcio total normales en un individuo se estiman entre 8,5 y 10,2 $\mathrm{mg} / \mathrm{dl}(2,12$ a $2,55 \mathrm{mmol} / \mathrm{L})$. Con mayor frecuencia se presentan parestesias periorales y en extremidades, calambres, mialgias y debilidad muscular ${ }^{(6,8)}$. Otros menos frecuentes son cólicos intestinales y disfagia por espasmos del músculo liso intestinal o esteatorrea que puede llevar a disfunción exocrina bilio-pancreática debido a insuficiencia en la secreción de colecistoquinina por la mucosa duodenal ${ }^{(9)}$. En el sistema respiratorio puede haber broncoespasmos y a nivel genitourinario y disfunción del detrusor. En las mujeres embarazadas con hipocalcemia se ha visto aumento en el parto pretérmino ${ }^{(6,10)}$. A nivel cardiovascular, el resultado varía desde bradicardia, angina, elevación de enzimas cardiacas, arritmias ventriculares, colapso cardiaco e hipotensión refractaria, hasta disfunción de contractilidad, lo que se evidencia en el electrocardiograma por intervalos QT prolongados, inversión de la onda T o hallazgos que simulan infarto y falla cardiaca ${ }^{(11)}$.

En el caso de niveles de calcio bajos de forma crónica, los pacientes pueden no presentar ningún síntoma y ser diagnosticados de forma incidental. Se ha asociado con anormalidades ectodérmicas, con síntomas poco perceptibles que van desde piel reseca hasta dermatitis, alopecia e incluso anormalidades dentales. El déficit neurológico constituido por extrapiramidalismo parecido a un cuadro de Parkinson y las cataratas subcapsulares pueden ser otras manifestaciones entre las descritas en hipocalcemia crónica ${ }^{(6)}$

Debido a esto y a la falta de información local, se realizó un estudio observacional en la Fundación Santa Fe de Bogotá, con el objetivo de presentar la epidemiología de la hipocalcemia posoperatoria en el manejo quirúrgico de cáncer de tiroides.

\section{Métodos}

Se realizó un estudio observacional de tipo corte transversal con la información obtenida de las historias clínicas de pacientes sometidos a cirugía de cuello desde el año 2003 hasta el 2017 en la Fundación Santa Fe de Bogotá. Los datos se obtuvieron a través de la base de datos realizada de manera prospectiva, que contó con variables demográficas, antecedentes patológicos, tipo de procedimiento y exámenes paraclínicos (PTH intacta, PTHi), calcio sérico, 25-OH vitamina D, fósforo sérico, magnesio sérico) de los pacientes operados por un cirujano de cabeza y cuello de la institución, con amplia experticia (cirujano de alto volumen); se verificó toda la información de variables y se completaron los datos faltantes, revisando una por una las historias clínicas de los pacientes.

Los resultados de laboratorio se recolectaron directamente del resultado oficial del laboratorio y el tipo de procedi- 
miento se confirmó con la nota quirúrgica de cada paciente. Todos los estudios de laboratorio de los pacientes fueron realizados del mismo laboratorio de la institución y se confirmó uso de la misma técnica y puntos de corte en todos los casos.

Se incluyeron pacientes mayores de 18 años sometidos a cirugía de cuello para manejo de cáncer de tiroides o sospecha de este diagnóstico (Bethesda IV, V y VI) en la Fundación Santa Fe de Bogotá con reportes documentados directamente del reporte del laboratorio de calcio sérico ionizado a los 3 y 7 meses posoperatorios. Se excluyeron pacientes con falla renal crónica, uso de PTH recombinante 1-34 para manejo de osteoporosis y pacientes con diagnóstico de hiperparatiroidismo.

De la base de datos inicial de 3.200 pacientes de cirugía de cuello, 1.200 se escogieron por diagnóstico citológico prequirúrgico de sospecha de cáncer de tiroides (Bethesda IV, V y VI). De este grupo, solo 640 fueron elegibles, se excluyeron pacientes por falta de datos de PTHi posoperatoria, calcio sérico en el posoperatorio o imposibilidad de confirmación de los datos, contando al final de la recolección con 261 pacientes a los cuales se les realizó el análisis de hipocalemia a los 3 y 7 meses posoperatorios. Todos los pacientes recibieron por protocolo suplencia de carbonato de calcio $500 \mathrm{mg}$ VO cada 8 horas y calcitriol 0,25 mcg cada 12 horas.

Se definieron como hipoparatiroidismo PTHi $<15$ pg/ml y calcio ionizado $<1,15 \mathrm{nmol} / \mathrm{l}$, e hipoparatiroidismo permanente la persistencia de estos valores con requerimiento de suplencia de calcio y calcitriol luego de siete meses del posoperatorio.

Se dividieron los pacientes en tres grupos, grupo A: aquellos con valores normales de calcio y PTHi a los tres y siete meses; grupo B: con hipocalcemia a los tres meses y normocalcemia a los siete meses posoperatorios; y grupo $\mathrm{C}$ con hipocalcemia a los tres meses y persistencia de calcio bajo a los siete meses.

En todos los pacientes con PTHi >15 pg/ml se intentó suspensión de calcio a los siete meses para evaluar respuesta; en aquellos con hipocalcemia persistente se reinició la suplencia de calcio y calcitriol.

El análisis de datos se hizo a través del programa estadístico SPSS versión 23 y Epidat 3.1, obteniendo frecuencias y porcentajes para las variables cualitativas y medidas de tendencia central y de dispersión para las cuantitativas; el análisis bivariado se realizó por sexo y valores séricos de control de calcio y PTH a los tres y siete meses.

\section{Resultados}

Se analizó un total de 261 pacientes. En el análisis univariado se evidenció un mayor porcentaje de mujeres (75\%) (figura 1); la mediana de la edad fue 48,6 años con un rango intercuartil de 14,1 (figura 2).

Los datos estadísticos del diagnóstico posoperatorio reflejan que el 85\% de los pacientes tenían diagnóstico de carcinoma papilar; $5 \%$, bocio multinodular; $2,7 \%$, adenoma folicular;
Figura 1. Sexo

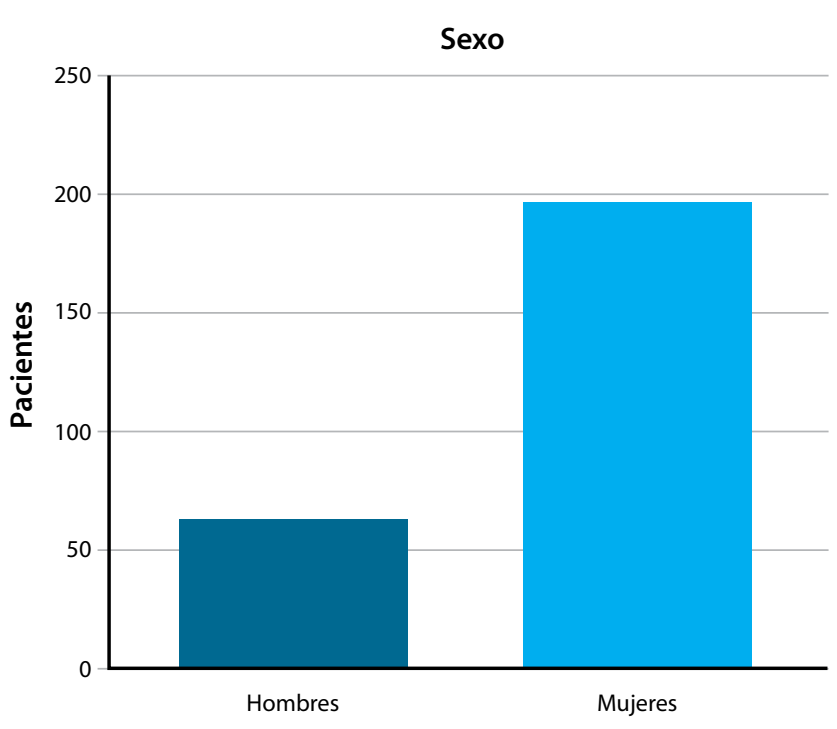

Figura 2. Edad

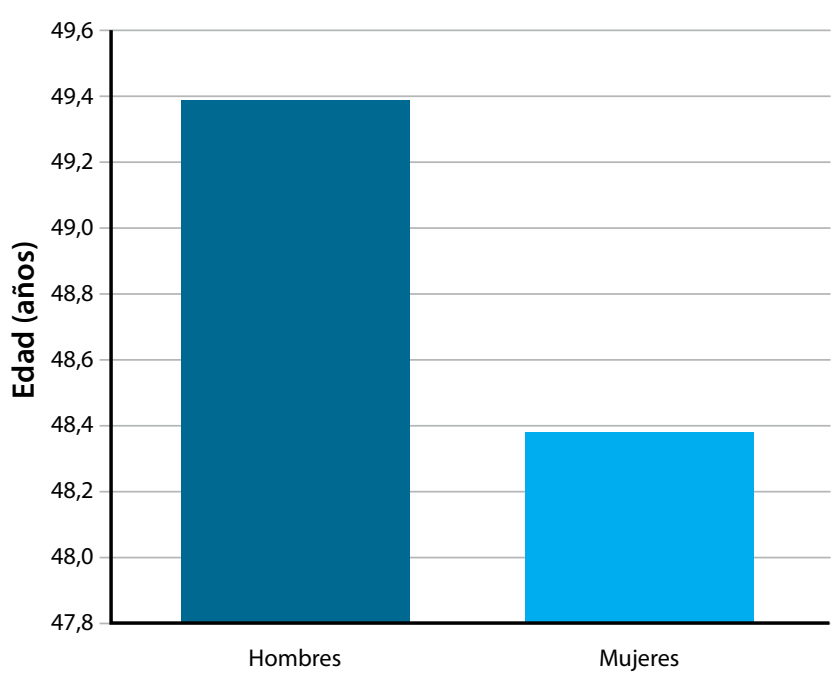

$2,3 \%$, bocio; $1,5 \%$, tiroiditis; $1,1 \%$, carcinoma medular; $1,1 \%$, carcinoma folicular; $0,4 \%$, adenoma de células de Hürtle; y 0,4\%, carcinoma anaplásico (figura 3 ).

El procedimiento que se realizó con mayor frecuencia fue tiroidectomía total más vaciamiento ganglionar central $(60,2 \%)$, seguido de tiroidectomía total $(32,2 \%)$, tiroidectomia total más limpieza paratraqueal $(6,9 \%)$ y hemitiroidectomía $(0,8 \%)$ (figura 4).

En el análisis bivariado se dividió a los pacientes en dos grupos según el valor de calcio iónico y PTH, a los tres y a los siete meses para determinar hipocalcemia posquirúrgica, siendo más prevalente a los tres meses posteriores con $18 \%$ 
Figura 3. Diagnóstico

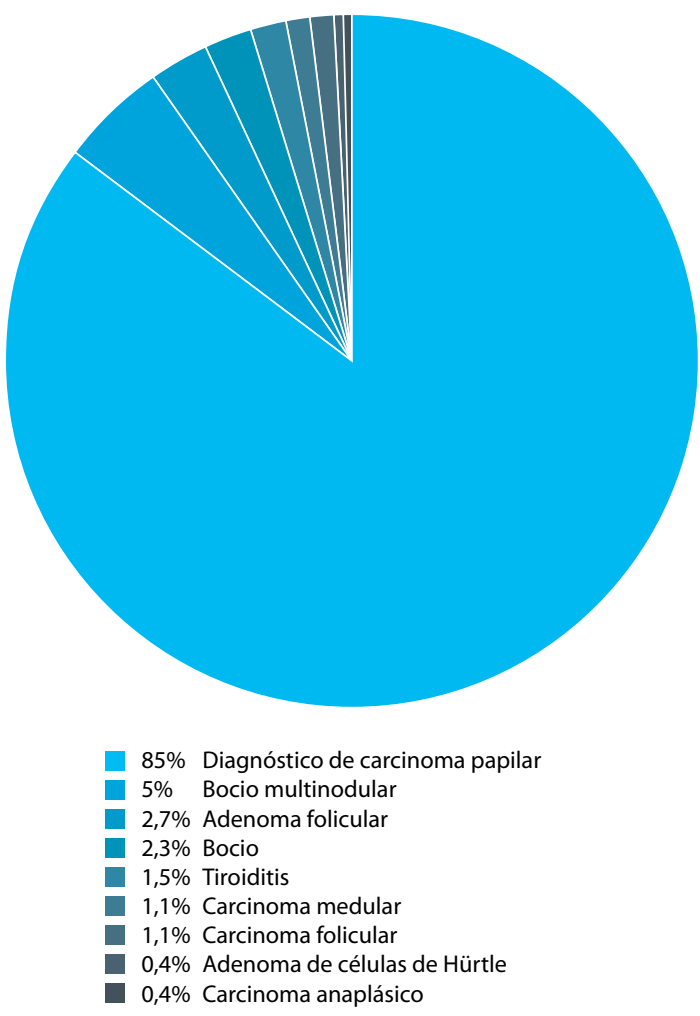

Figura 4. Procedimiento

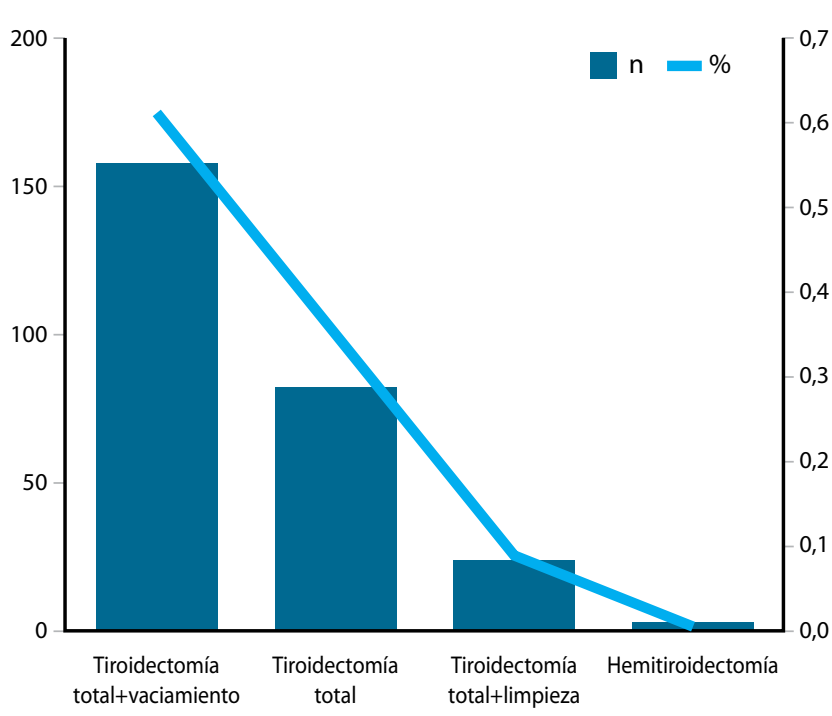

de los pacientes afectados, frente a $7 \%$ a los siete meses como desenlaces finales (figuras $\mathbf{5}$ y $\mathbf{6}$ ).

El procedimiento con mayor asociación a hipoparatiroidismo fue la tiroidectomía total + vaciamiento central en $62 \%$ de los casos de hipocalcemia transitoria y $65 \%$ de permanente. El diagnóstico más relacionado fue carcinoma papilar de tiroides que ocupó el $85 \%$ de casos de hipocalcemia transitoria y el
95\% de permanente. Por el contrario, en patología benigna solo se presentaron tres casos de hipoparatiroidismo transitorio y uno permanente. Solo 33 de los 47 pacientes con hipocalcemia a los tres meses tenían PTHi menor de 15 pg/ml y 15 de los 19 casos con hipocalcemia a los siete meses mantenían PTHi baja.

Estos casos de hipocalcemia con PTHi normal se han descrito en varias revisiones, más aún, guías internacionales y revisiones de la literatura publicadas recientemente sugieren contar los casos de hipocalcemia transitoria o permanente con niveles de PTHi normal dentro de la prevalencia de hipocalcemia posoperatoria para evitar subdiagnósticos, subregistros y complicaciones futuras ${ }^{(12,13)}$.

Ninguno de los pacientes presentó reingresos tempranos (primeras dos semanas del posoperatorio) por hipocalcemia. Por referencia del grupo de cirugía y endocrinología, estos

Figura 5. Hipocalcemia POP tres meses

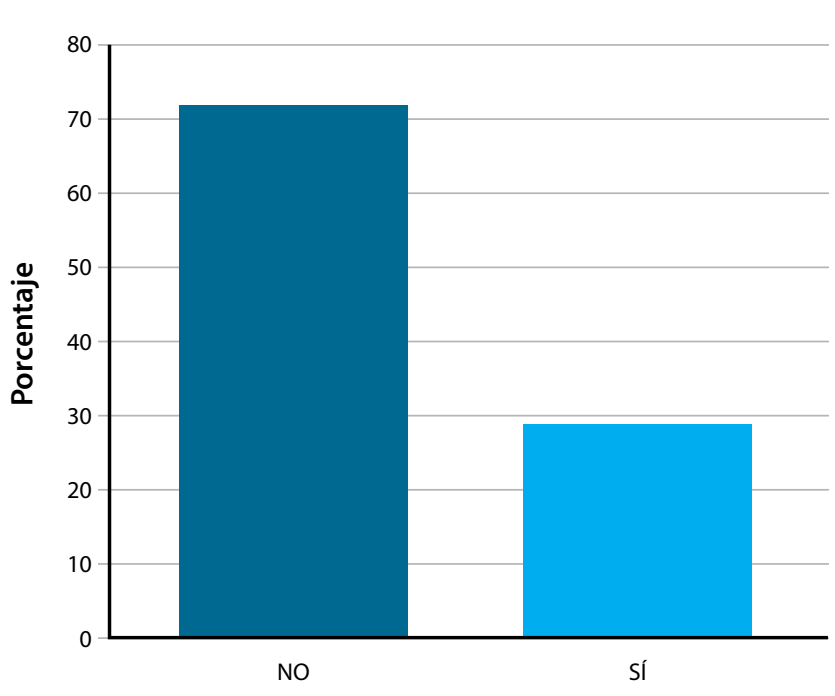

Figura 6. Hipocalcemia POP siete meses

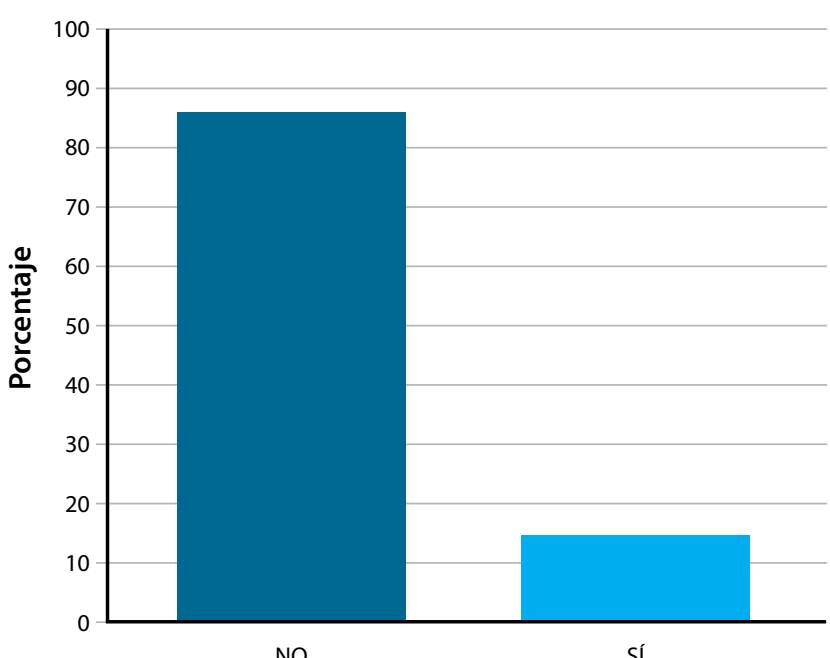

NO

sí 
reingresos tempranos sí se presentaban antes de la implementación de suplencia posoperatoria inmediata de calcio y calcitriol a todos los pacientes después de cirugía de cuello.

\section{Discusión}

La hipocalcemia por hipoparatiroidismo es la complicación más frecuente de la cirugía de tiroides ${ }^{(14-16)}$, esta condición genera severas alteraciones en la calidad de vida con síntomas en muchos casos incapacitantes, así como incremento en los gastos y costos del paciente, de la familia y del sistema de salud por ausentismo laboral y necesidad de múltiples ingresos a servicios de urgencias ${ }^{(17-19)}$, muchos casos se mantienen asintomáticos con hipocalcemia crónica que puede desencadenar complicaciones a largo plazo por precipitación de fosfatos de calcio $^{(20,21)}$.

El hipoparatiroidismo transitorio se refiere a la presencia de hipocalcemia y niveles bajos de PTH los primeros siete meses después de la cirugia y permanente si persiste después del sexto mes posquirúrgico como fue definido por la ATA (Asociación Americana de Tiroides) en reciente publicación ${ }^{(12)}$. La incidencia reportada varía entre los estudios; en hipoparatiroidismo transitorio entre $19 \%$ y $38 \%$, y permanente entre $0 \%$ y $33 \%$ con grandes diferencias en estos valores, incluso en recientes metanálisis y revisiones sistemáticas ${ }^{(3,22)}$. Estas diferencias parecen provenir de la institución, la experticia del cirujano, los diagnósticos prequirúrgicos, la práctica rutinaria de autoimplante de paratiroides, la presencia o ausencia de PTHi baja en casos de hipocalcemia persistente y la divergencia entre estudios en el tiempo de permanencia tomado para definir hipoparatiroidismo permanente con puntos de corte de siete meses vs. un año ${ }^{(12,23,24)}$.

El diagnóstico más freceuentemente asociado a hipoparatiroidismo posquirúrgico ha sido la presencia de bocio muy grande, enfermedad de Graves-Basedow y patología maligna y los procedimientos más relacionados son tiroidectomía total con vaciamiento central y reintervenciones ${ }^{(3,23,25-27)}$; sin embargo, se debe aclarar que la tiroidectomía como manejo de patología benigna se realiza cada vez con menor frecuencia y la técnica quirúrgica ha mejorado con los años. En nuestro estudio se evaluaron únicamente los pacientes llevados a tiroidectomía con o sin vaciamiento ganglionar para manejo de sospecha de cáncer de tiroides; los casos de patología final benigna presentaron menores tasas de hipocalcemia como lo reporta la literatura; sin embargo, estos casos también se trataron de manera menos agresiva, usualmente con tiroidectomía sin vaciamiento.

Algunos grupos de grandes instituciones en el mundo han propuesto protocolos tanto de prevención como de manejo temprano para reducir la incidencia de hipocalcemia simtomática posoperatoria ${ }^{(23,28-30)}$. A la fecha no disponemos en nuestro país de estudios de prevención y manejo temprano de hipocalcemia posoperatoria, dado que no se encuentra tampoco reportada la prevalencia de esta complicación en Colombia. En este estudio presentamos la prevalencia de hipocalcemia en una institución de cuarto nivel de complejidad en pacientes operados por un cirujano de alto volumen con un protocolo de suplementación de calcio y calcitriol a todos los pacientes sometidos a cirugía de cuello, como lo realizan algunos grupos que han demostrado la reducción de ingresos tempranos al servicio de urgencias luego del inicio de la suplementación obligatoria de calcio y calcitriol después de la cirugía.

En este estudio las tasas de la complicación fueron menores que las reportadas en la literatura, se considera que esto se debe a que los procedimientos fueron realizados en una institución de cuarto nivel de complejidad, por un cirujano experto y todos los pacientes recibieron suplementación desde el posoperatorio inmediato. A pesar de las mejores condiciones en el manejo quirúrgico, el hipoparatiroidismo posoperatorio es una complicación posible que debe manejarse idealmente de forma temprana y seguirse en el tiempo.

Una de las grandes limitaciones del estudio fue la pérdida de datos de algunas variables, pasando de un elevado volumen de pacientes 1200 a 261, lo cual se sugiere que podría cambiar los datos de prevalencia. Disponemos de la información verbal clara del grupo de endocrinólogos, urgenciólogos y cirujanos de la institución que aseguran que los reingresos a urgencias por hipocalcemia de los pacientes sometidos a cirugías de compartimento central del cuello cesaron cuando se inició la suplementación obligatoria, pero no contamos con un grupo de comparación antes y después de la implementación de este protocolo de suplementación.

\section{Conclusiones}

Si bien la prevalencia de hipocalcemia posoperatoria en nuestra institución en condiciones ideales fue menor que la reportada en la literatura, esta es una complicación con tasas de prevalencia no despreciables que altera la calidad de vida de los pacientes. Es importante tener protocolos de prevención de las condiciones más asociadas a hipocalcemia posoperatoria. Iniciar la suplementación temprana que puede no reducir la aparición de hipoparatiroidismo permanente pero sí la morbilidad de la hipocalcemia y los reingresos a urgencias. Se requiere realizar más investigación en este campo en nuestro país y crear registros a nivel nacional que permitan analizar el comportamiento en general de los pacientes sometidos a cirugía de cuello, con el fin de establecer protocolos en la suplementación que prevengan el porcentaje de morbilidad de los pacientes con secuelas permanentes.

\section{Conflictos de interés:}

Ninguno.

Trabajo realizado en el Hospital Universitario Fundación Santa Fe de Bogotá, Calle 119 No. 7-75, Bogotá, Colombia 


\section{Referencias}

1. Clarke BL, Brown EM, Collins MT, Jüppner H, Lakatos P, Levine MA, et al. Epidemiology and Diagnosis of Hypoparathyroidism. J Clin Endocrinol Metab. 2016;101(6):2284-99.

2. De Sanctis V, Soliman A, Fiscina B. Hypoparathyroidism: from diagnosis to treatment. Curr Opin Endocrinol Diabetes Obes. 2012;19(6):435-42.

3. Edafe O, Antakia R, Laskar N, Uttley L, Balasubramanian SP. Systematic review and meta-analysis of predictors of post-thyroidectomy hypocalcaemia. Br J Surg. 2014;101(4):307-20.

4. Shoback DM, Bilezikian JP, Costa AG, Dempster D, Dralle H, Khan AA, et al. Presentation of Hypoparathyroidism: Etiologies and Clinical Features. J Clin Endocrinol Metab. 2016;101(6):2300-12.

5. Maeda SS, Fortes EM, Oliveira UM, Borba VC, Lazaretti-Castro M. Hypoparathyroidism and pseudohypoparathyroidism. Arq Bras Endocrinol Metabol. 2006;50(4):664-73.

6. Lee JW. Fluid and electrolyte disturbances in critically ill patients. Electrolyte Blood Press. 2010;8(2):72-81.

7. Patil NJ, Yadav SS, Gokhale YA, Padwa N. Primary hypoparathyroidism: psychosis in postpartum period. J Assoc Physicians India. 2010;58:506-8.

8. Al-Azem H, Khan AA. Hypoparathyroidism. Best Pract Res Clin Endocrinol Metab. 2012;26(4):517-22.

9. Abboud B, Daher R, Boujaoude J. Digestive manifestations of parathyroid disorders. World J Gastroenterol. 2011;17(36):4063-6.

10. Shoback D. Clinical practice. Hypoparathyroidism. N Engl J Med. 2008:359(4):391-403

11. Ilveskoski E, Sclarovsky S, Nikus K. Severe hypocalcemia simulating STelevation myocardial infarction. Am J Emerg Med. 2012;30(1):256.e3-6.

12. Orloff LA, Wiseman SM, Bernet VJ, Fahey TJ, Shaha AR, Shindo ML, et al. American Thyroid Association Statement on Postoperative Hypoparathyroidism: Diagnosis, Prevention, and Management in Adults. Thyroid. 2018;28(7):830-41.

13. Edafe 0, Balasubramanian SP. Incidence, prevalence and risk factors for post-surgical hypocalcaemia and hypoparathyroidism. Gland Surg. 2017;6(Suppl 1):S59-S68.

14. Bollerslev J, Rejnmark L, Marcocci C, Shoback DM, Sitges-Serra A, van Biesen W, et al. European Society of Endocrinology Clinical Guideline: Treatment of chronic hypoparathyroidism in adults. Eur J Endocrinol. 2015;173(2):G120.

15. Stack BC, Bimston DN, Bodenner DL, Brett EM, Dralle H, Orloff LA, et al. AMERICAN ASSOCIATION OF CLINICAL ENDOCRINOLOGISTS AND AMERICAN COLLEGE OF ENDOCRINOLOGY DISEASE STATE CLINICAL REVIEW: POSTOPERATIVE HYPOPARATHYROIDISM - DEFINITIONS AND MANAGEMENT. Endocr Pract. 2015;21(6):674-85.

16. Hauch A, Al-Qurayshi Z, Randolph G, Kandil E. Total thyroidectomy is associated with increased risk of complications for low- and high-volume surgeons. Ann Surg Oncol. 2014;21(12):3844-52.

17. Sikjaer T, Moser E, Rolighed L, Underbjerg L, Bislev LS, Mosekilde L, et al. Concurrent Hypoparathyroidism Is Associated With Impaired Physical Function and Quality of Life in Hypothyroidism. J Bone Miner Res. 2016;31(7):1440-8.

18. Arlt W, Fremerey C, Callies F, Reincke M, Schneider P, Timmermann W, et al. Well-being, mood and calcium homeostasis in patients with hypoparathyroidism receiving standard treatment with calcium and vitamin D. Eur Endocrinol. 2002;146(2):215-22.

19. Büttner M, Musholt TJ, Singer S. Quality of life in patients with hypoparathyroidism receiving standard treatment: a systematic review. Endocrine. 2017;58(1):14-20.

20. Underbjerg L, Sikjaer T, Mosekilde L, Rejnmark L. Cardiovascular and rena complications to postsurgical hypoparathyroidism: a Danish nationwide controlled historic follow-up study. J Bone Miner Res. 2013;28(11):2277 85.

21. Mitchell DM, Regan S, Cooley MR, Lauter KB, Vrla MC, Becker CB, et al. Long term follow-up of patients with hypoparathyroidism. J Clin Endocrinol Metab. 2012;97(12):4507-14.

22. Kakava K, Tournis S, Papadakis G, Karelas I, Stampouloglou P, Kassi E, et al. Postsurgical Hypoparathyroidism: A Systematic Review. In Vivo. 2016;30(3):171-9.

23. Mejia MG G-DD, Fierro F, Tapiero M, Rojas L and Cadena E. Hypocalcemia posthyroidectomy: prevention, diagnosis and management2018; 4(2):[1-7 pp.].

24. Ritter K, Elfenbein D, Schneider DF, Chen H, Sippel RS. Hypoparathyroidism after total thyroidectomy: incidence and resolution. J Surg Res. 2015;197(2):348-53.

25. Testini M, Gurrado A, Avenia N, Bellantone R, Biondi A, Brazzarola P, et al. Does mediastinal extension of the goiter increase morbidity of total thyroidectomy? A multicenter study of 19,662 patients. Ann Surg Oncol. 2011;18(8):2251-9.

26. Giordano D, Valcavi R, Thompson GB, Pedroni C, Renna L, Gradoni P, et al. Complications of central neck dissection in patients with papillary thyroid carcinoma: results of a study on 1087 patients and review of the literature. Thyroid. 2012;22(9):911-7.

27. Ito Y, Kihara M, Kobayashi K, Miya A, Miyauchi A. Permanent hypoparathyroidism after completion total thyroidectomy as a second surgery: How do we avoid it? Endocr J. 2014;61(4):403-8.

28. Alía P, Moreno P, Rigo R, Francos JM, Navarro MA. Postresection parathyroid hormone and parathyroid hormone decline accurately predict hypocalcemia after thyroidectomy. Am J Clin Pathol. 2007;127(4):592-7.

29. Bellantone R, Lombardi CP, Raffaelli M, Boscherini M, Alesina PF, De Crea $\mathrm{C}$, et al. Is routine supplementation therapy (calcium and vitamin D) useful after total thyroidectomy? Surgery. 2002;132(6):1109-12; discussion 12-3.

30. Carter Y, Chen H, Sippel RS. An intact parathyroid hormone-based protocol for the prevention and treatment of symptomatic hypocalcemia after thyroidectomy. J Surg Res. 2014;186(1):23-8. 